\title{
Probing the strain distribution within a single crystal superalloy during high temperature testing
}

\author{
Alain Jacques ${ }^{1, \text { a }}$, Mohamed Biskri ${ }^{1}$, Thomas Schenk ${ }^{1}$, Jean Philippe Chateau Cornu ${ }^{1}$, and Pierre Bastie ${ }^{2}$ \\ ${ }^{1}$ Institut Jean Lamour (UMR CNRS-UL N ${ }^{\circ}$ 7198), Labex DAMAS, Parc de Saurupt, 54000 Nancy, France \\ ${ }^{2}$ LiPhy, 140 Avenue de la Physique, BP. 87, 38402 Saint-Martin-d'Hères, France
}

\begin{abstract}
The combination of in situ high resolution diffraction experiments with synchrotron radiation and mechanical and diffraction modelling is used to investigate the microstructure and mechanical state of single crystal superalloys during high temperature tests: a very high temperature annealing without external load and a creep test.
\end{abstract}

\section{Introduction}

Single crystal superalloys are both strategic industrial materials and model materials to understand and predict the plastic behaviour of polyphased materials. As the conversion efficiency of thermal motors such as turboreactors and gas turbines is linked to their operating temperatures, the ability to manufacture the components of their hottest parts (high pressure turbine blades and vanes) and to predict their behaviour and life time under various conditions from normal use to extreme events is of major industrial interest.

Besides their industrial importance, single crystal superalloys have either a cuboid microstructure, or a socalled rafted microstructure: they are made of coherent cuboids or of semi coherent platelets of hard $\mathrm{L}_{2} \gamma^{\prime}$ phase perpendicular to the [001] tensile axis [1,2] embedded within a ductile fcc $\gamma$ matrix. Differences between the mechanical behaviour of the two phases and a lattice mismatch of a few $10^{-3}$ generate internal stresses which superimpose to the applied load. At high temperature, the $\gamma$ matrix deforms by glide of perfect $\mathrm{a} / 2<110>$ dislocations, while the plastic strain of the $\gamma^{\prime}$ phase is believed to occur by climb of $a^{\prime}<100>$ and/or a' $<110>$ dislocations exchanging vacancies [3].

In order to build physically (i.e. dislocation) based constitutive laws not only for the composite material, but for each phase, macroscopic data such as the applied stress and the average strain rate are insufficient: we need at least to know the average stress (strain) state of each phase, or better the distribution of stresses (strains) within each phase. We also need a tool to check the validity of such laws. The aim of the present paper is to show that the combination of in situ experiments using high resolution synchrotron X-Ray diffractometry and diffraction peak modelling can be such a tool.

${ }^{a}$ Corresponding author: alain.jacques@univ-lorraine.fr

\section{Experiments}

Figure 1 shows the layout of a Three Crystal Diffractometer [4]. The initial polychromatic X-Ray beam first goes through a (311) silicon monochromator. The monochromatized beam is then diffracted by the sample ((200) diffracting vector): different areas in Bragg conditions with slightly different lattice parameters scatter the beam into slightly different directions. A second (311) silicon crystal called analyser diffracts again one of those beams into an energy sensitive Ge detector. A rotation of the analyser probes the distribution of the beams scattered by the specimen, i.e. the distribution of interplanar distances within the specimen, while a rotation of the specimen measures the distribution of orientations of the diffracting planes. As the (311) reflection of silicon is very narrow, the "instrumental width" of the device is lower than a second of arc for a $3.65^{\circ}$ two theta angle and can be neglected. A further reduction of the background can be obtained by use of a double crystal diffractometer.

Using the high energy beam of the ID 15 beamline of the ESRF or the P07 beamline of the PETRA III ring at Hasylab (between $100 \mathrm{keV}$ and $150 \mathrm{keV}$ ), it is possible to do experiments in transmission and to measure lattice parameters in the bulk of the specimens. Thanks to the high intensity of the synchrotron beam, a 200 points scan can be recorded in less than 5 minutes: it is possible to study the response of the material at short time intervals following a change of the applied load or temperature.

\section{Scattering by a strained material}

The shape of a diffraction peak depends on the distribution of lattice strains within the material and the size of the coherent zones. If we assume that the coherence length of $\mathrm{X}$-Rays is larger than a few micrometres, the amplitude scattered in the vicinity of a vector $\mathbf{G}$ of the reciprocal lattice along a vector $\mathbf{q}=\mathbf{G}+\mathbf{q}^{\prime}$ can be written as:

$$
A\left(\boldsymbol{q}^{\prime}\right)=F T\{f(x) \cdot \exp [2 i \pi \boldsymbol{G} \cdot \boldsymbol{u}(x)]\} .
$$

This is an Open Access article distributed under the terms of the Creative Commons Attribution License 4.0, which permits unrestricted use, distribution, and reproduction in any medium, provided the original work is properly cited. 


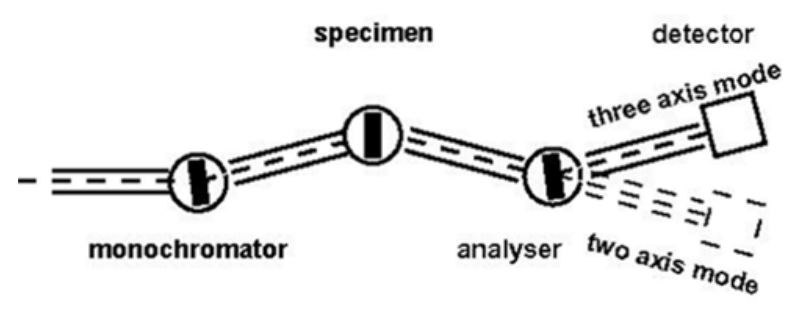

Figure 1. Three Crystal Diffractometer.

Where FT indicates the Fourier Transform, $\mathrm{f}(\mathrm{x})$ is the local scattering factor (depending on the local chemistry and local structure amplitude), and $\mathbf{u}(\mathrm{x})$ is the displacement field within the material [5]. The diffracted intensity can then be obtained by taking $\mathrm{I}=\mathrm{A} . \mathrm{A}^{*}$, where $\mathrm{A} *$ is the complex conjugate of A.

If the displacement field can be calculated, it is thus possible to generate a theoretical diffraction peak which may be compared to an experimental one. Various methods can be used:

- Direct calculation of the superposition of the displacement fields of individual cuboidal inclusions.

- Finite element methods.

- Fourier transform methods [6].

While the first two methods are computer intensive and, in the first case, require analytical formulas, Fast Fourier Transform (FFT) methods are quite efficient for large numbers of voxels. (The same calculation for a $128^{3}$ volume takes 23 hours on a desktop computer with the first method, and 12 minutes with the third.) Furthermore, FFT calculations can be extended easily for inhomogeneous materials with anisotropic elastic properties [7].

Figure 2 a shows a first example of the displacement field $U_{x}$ calculated in such a way for a $2 \mu \mathrm{m}$ cube of superalloy containing a 0.7 volume fraction of precipitates (size approx. $0.45 \mu \mathrm{m}$. See dotted lines) and a -0.003 lattice mismatch. The corresponding peak, and a top view of its enlarged tail are given in Figs. $2 b$ and $2 c$, while Fig. 2 d shows the TCD profiles (vertically offset) expected for the same cuboids microstructure, but with cubes having bevelled edges and corners. While for perfect cubes, a low and wide peak is expected for a 0.0045 strain, a narrower but taller peak is obtained at 0.002 for a $0.1 \mu \mathrm{m}$ bevel. Assuming a constant volume fraction 1-f of $\gamma$ matrix, a large bevel results in more $\gamma$ phase at the edges (circle in Fig. 2a) and corners, and less in the corridors (rectangle) which become thinner: the intensity scattered by the corridors is lower and distributed on a wider peak. The main peak also becomes slightly thinner as the highly stressed elastic singularities at the corners of the cubes disappear. Last, the distribution of cuboid sizes is wider in a real microstructure than in the present model.

\section{Spontaneous loss of coherence during high temperature annealing}

\subsection{Experimental results.}

Figure 3 shows the evolution of the high resolution (200) diffraction peak of a specimen with a cuboid
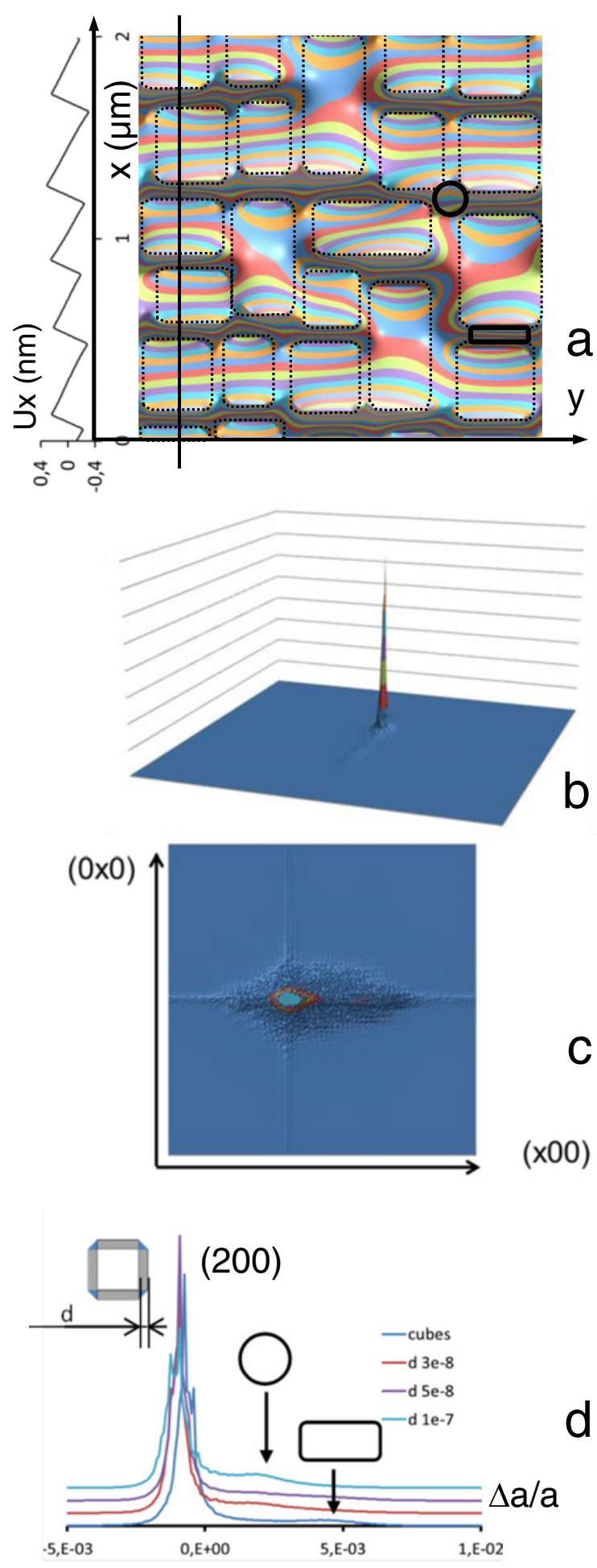

Figure 2. yx plot of the displacement field in the $\mathrm{x}$ direction in a superalloy and profile along the straight vertical line (a), and (200) diffraction peaks (b,c,d). See text for details.

microstructure during a high temperature annealing at $1130{ }^{\circ} \mathrm{C}$

The top of the peak (Fig. 3a) was recorded with a 1 second counting time per step, while a 5 seconds recording and averaging were used for the tails (Logarithmic scale, Fig. 3b. All diffraction profiles were normalized to a constant unit area for comparison. The peak intensity 

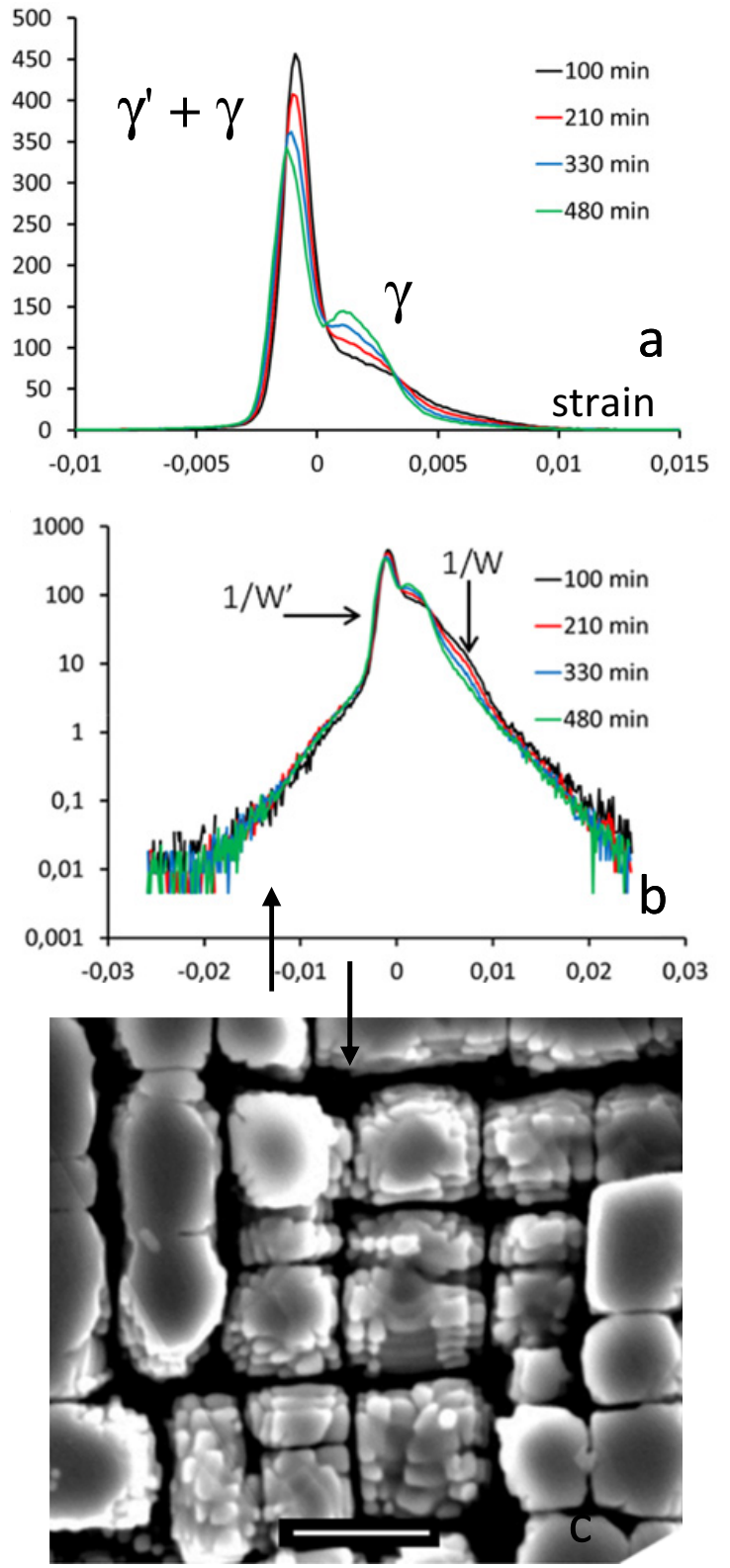

Figure 3. High resolution diffraction peak of a superalloy at $1130^{\circ} \mathrm{C}$ in linear (a) and logarithmic (b) scale. SEM micrograph (1 $\mu \mathrm{m}$ mark) after chemical etching.

at the top is 500 (arbitrary units) and the tails level at 0.01: the peak to background ratio is close to the $10^{5}$ range. The main peak corresponds to the $\gamma^{\prime}$ cuboids with a contribution of the $\gamma$ corridors parallel to the (200) diffracting vector. The tail on the large parameters side (Fig. 3a, right) is due to the $\gamma$ corridors perpendicular to (200) [8]. The long range tails nearly follow an $\varepsilon^{-4}$ law: this is probably Huang scattering resulting from the highly strained zones on the vicinity of point defects. At intermediate strains, an interesting feature of the peaks is their exponential tail (i.e. their linear slope when seen with a logarithmic scale). The inverses of these slopes are defined as the $\mathrm{W}$ and $\mathrm{W}$ ' parameters which will be discussed below.

During annealing, the width of the main $\gamma^{\prime}$ peak and its $\gamma$ tail decreased progressively, and the height of the $\gamma^{\prime}$ peak decreased. Another wider peak appeared at strain 0.0015. The height of the left tail (Logarithmic scale) was unchanged, while it slightly decreased on the right side.

After cooling, polishing and chemical etching $(66 \%$ $\mathrm{HCl}$ et $33 \% \mathrm{HNO}_{3}$ ), the specimens were observed by SEM. Lines corresponding to dislocation traces can be seen at the surface of the cuboids, with an average distance of about $0.02 \mu \mathrm{m}$. During the high temperature annealing, the $\gamma^{\prime}$ volume fraction was lower than at room temperature, and the $\gamma$ corridors wider. The magnitude of the lattice mismatch was larger than 0.003, and the coherence stresses became larger than the Orowan stress, allowing dislocations to glide into the $\gamma$ corridors and partly release the coherence stresses. As this plastic relaxation allowed the lattice parameter of the $\gamma$ corridors parallel to (200) to increase in the [100] direction, the corresponding peak shifted to the right. The intensity scattered at the position of the $\gamma^{\prime}$ peak thus decreased. The high lattice parameter of the corridors perpendicular to (200) was due to the coherence stresses in the (100) plane: as these stresses decreased, the position of the peak shifted to the left.

\subsection{Peak modelling}

Figure 4a shows a simulation of the diffraction peak (thin black line) for comparison with the experimental peak recorded at 100 minutes (Fig. 3a). The displacement field was calculated in a $2 \mu \mathrm{m}^{3}$ representative volume containing 64 cuboids with a random size and position (0.42 volume fraction of $\gamma^{\prime}$ phase), and assuming a -0.0035 lattice mismatch between both phases. By arbitrarily giving a 0 and 1 scattering amplitude to the $\gamma^{\prime}$ (blue) and the $\gamma$ (red) phases, it is possible to calculate their contributions to the peaks. The $\gamma^{\prime}$ peak contributes to the main peak only, while the $\gamma$ intensity is distributed between the main peak (coherent corridors parallel to the $\mathrm{g}$ vector), the highly strained corridors perpendicular to the edges (rectangles in Fig. 2a) and the cube edges (circles). A $4 \cdot 10^{-8}$ bevel at the cubes corners and edges was assumed: this is probably an underestimated value, as the area of the intermediate peak is lower than the experimental one, and the right peak higher. Last, the width of the calculated intermediate peak is lower than the experimental one.

Figure $4 \mathrm{~b}$ compares the (green) experimental peak recorded at 480 minutes and peaks simulated assuming that the lattice mismatch had been half relaxed by infinitesimal dislocations. Both $\gamma$ and $\gamma^{\prime}$ peaks are thinner than in the unrelaxed microstructure (their width is approximately divided by two) and their positions have been relatively shifted by 0.00175 , i.e. the plastic strain. However, if the calculated peaks have correct positions, they are thinner than the experimental ones: an ingredient is missing in the calculation. We believe this fact results from the choice to use infinitesimal dislocations instead of perfect dislocations of the matrix: as the lattice planes remain continuous between interface dislocations, there is no jump of the lattice parameters and some intensity should be diffracted between the two peaks of Fig. $4 \mathrm{~b}$. 

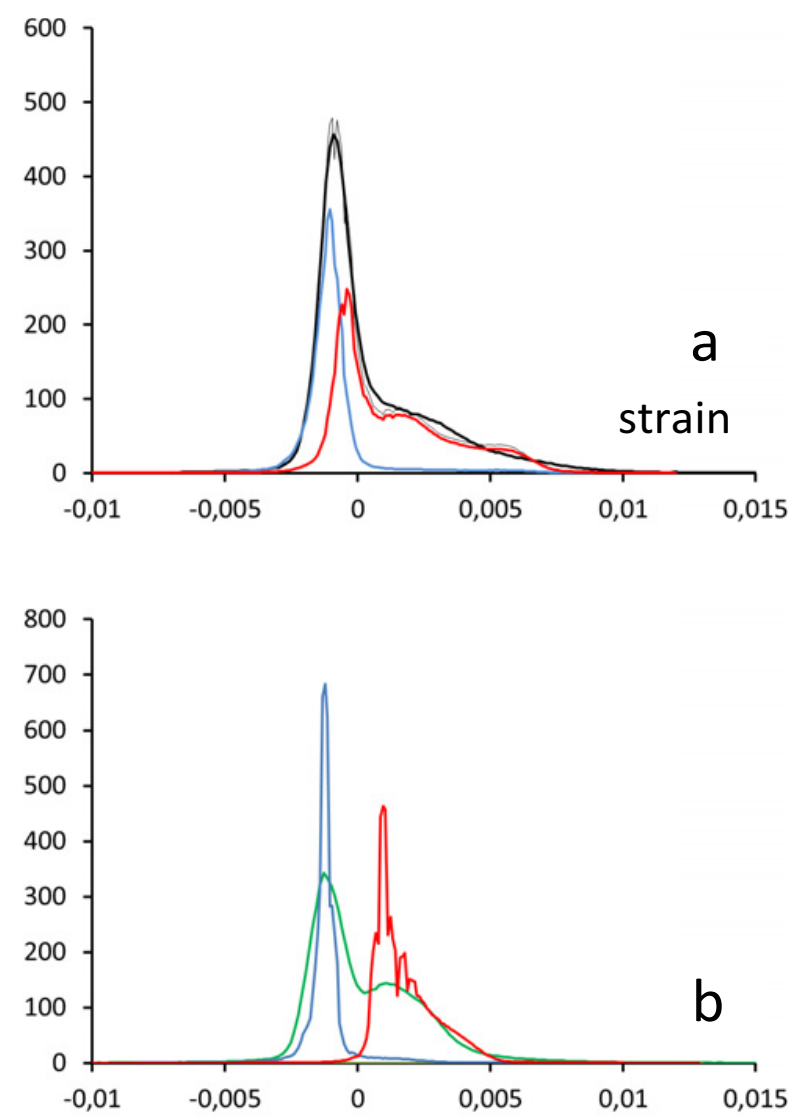

Figure 4. Simulated high resolution diffraction peak of a superalloy with an unrelaxed (a) and half relaxed (b) microstructure.

\section{Peak widening during creep}

\subsection{Experimental results}

Figure 5 shows the evolution of the $\mathrm{W}$ and $\mathrm{W}$ ' parameters of the diffraction peaks of a rafted superalloy during a tensile creep test under variable load at $1080^{\circ} \mathrm{C}$ [9]. The applied load $\sigma_{a}$ and the strain rates of each phase $(\gamma$ : thin line and $\gamma^{\prime}$ : thick line) are given for comparison.

The $\mathrm{W}$ parameter of the $\gamma$ peak remains constant during the beginning of the test, and only begins to increase after a jump of the applied load up to $200 \mathrm{MPa}$. After partial unloading of the specimen at $\mathrm{t}=55000 \mathrm{~s}$, it returns to its initial value. The W' parameter is initially $3.510^{-4}$. It begins to increase at $35000 \mathrm{~s}$, under a $150 \mathrm{MPa}$ applied load, when the strain rate of the $\gamma$ phase is in the $10^{-8} \mathrm{~s}^{-1}$ range. It increases up to $5.510^{-4}$ at the maximum load, then decreases to $4.210^{-4}$ after unloading.

In the W' vs. W plot of Fig. 5b, the experimental points initially move from $\mathrm{A}$ to $\mathrm{B}$, then move to the top and downwards along the straight $\mathrm{BC}$ line. The slope of $\mathrm{BC}$ varies with temperature in the $980{ }^{\circ} \mathrm{C}$ to $1130{ }^{\circ} \mathrm{C}$ range. Under high stresses, the evolutions of $\mathrm{W}$ and $\mathrm{W}^{\prime}$ are thus correlated, and are believed to be related to the dislocation density within the $\gamma^{\prime}$ phase. This may be checked in Fig. 5c, which shows a $2.510^{12} \mathrm{~m}^{-2}$ dislocation density in a TEM micrograph of the same specimen after cooling under high load, as the (W, W') point was at C.

The same behaviour was observed on all specimens strained at temperatures higher than $980^{\circ} \mathrm{C}$.
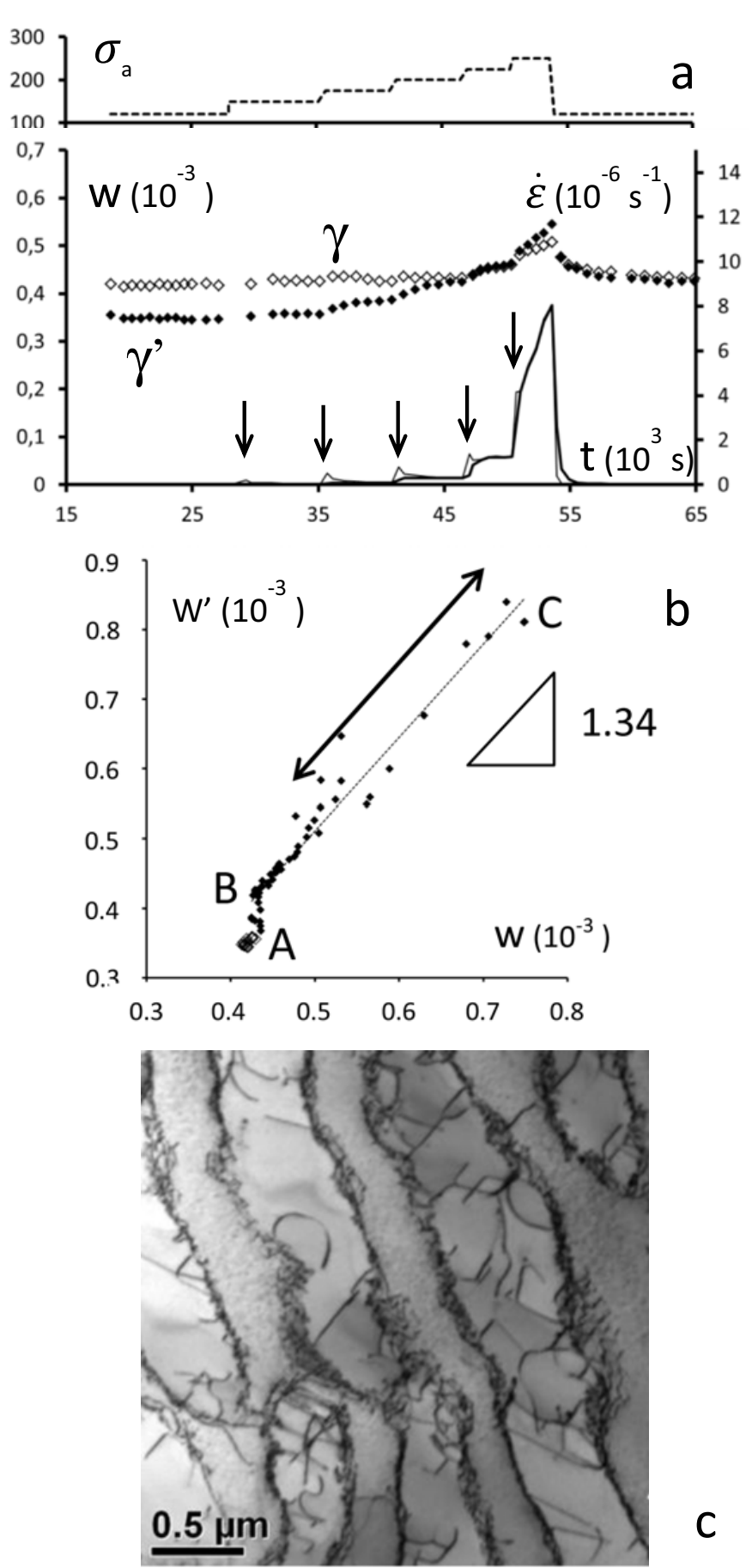

Figure 5. a) Plot of the variations with time (1000 s) of the applied load (top), the W (empty diamonds) and W'(full diamonds) fit parameters $\left(10^{-3}\right.$ strain units, bottom left hand scale) and the strain rate of each phase (bottom right hand scale). b) Plot of W' vs. W. c) Post mortem TEM micrograph taken after cooling of the specimen under high stress.

\subsection{Local stresses and long range stresses}

\subsubsection{Long range stresses}

Figure 6a schematizes the distribution of stresses within the rafted microstructure at the beginning of the second 
stage of a creep curve. During stage I, dislocations moved within the (clear) $\gamma$ corridors between (dark) $\gamma^{\prime}$ rafts, and left dislocation segments at the $\gamma / \gamma^{\prime}$ interfaces. If given time, these segments may react to form new dislocation segments with their Burgers vector perpendicular to the [001] tensile axis, and form a more or less relaxed dislocation wall. The stress state of matter within the microstructure is then the sum of the applied load $\sigma_{z z}=\sigma_{a}$, and of coherence stresses along the $\mathrm{x}$ and $\mathrm{y}$ directions due to the lattice mismatch $\delta$ and the stress field of the interfacial dislocation walls.

It has been shown in [9] that the average resolved shear stress for the plasticity of the $\gamma$ corridors is equal to the average Orowan stress $\sigma_{O}$. We may admit this remains true for each corridor: new dislocations may enter a corridor as soon as the local Von Mises stress $\sigma_{V M}=\sigma_{z z}-\sigma_{x x}$ is equal to the Orowan stress for this corridor. These dislocations will stop entering as soon as the increased stress field of the dislocation walls at the interfaces between the same corridor and its neighbouring $\gamma^{\prime}$ rafts has become large enough to repel them. We can thus expect each corridor to have on its both interfaces dislocation walls with the same dislocation density. On the contrary, different corridors with different thicknesses and different Orowan stresses will have different interfacial dislocation densities. From this, we can expect a heterogeneous distribution of internal stresses in the $\gamma$ phase (i.e. a wide $\gamma$ peak), and a homogenous distribution of stresses in the $\gamma^{\prime}$ rafts, because the stress fields of the interface dislocations of the corridor between two neighbouring rafts will cancel.

When the applied load is high enough, the $\gamma^{\prime}$ rafts begin to deform plastically. As in the case of corridors, new dislocations will be added at the interfaces on both sides of a raft, or will cancel with existing ones. If the plastic strain is the same in all rafts, the same number of dislocations will disappear on both sides of all interfaces, and the distribution of stresses will remain the same. Conversely, if some rafts deform more or less than others (Fig. 6b), the density of dislocations on both sides of a corridor will become different, and the stress fields of both interfaces will no longer cancel: the internal stresses within the $\gamma^{\prime}$ phase will become heterogeneous, and the width of the $\gamma^{\prime}$ peak will increase.

\subsubsection{Dislocations and reversible changes in the shapes of the diffraction peaks}

While the contribution of dislocations has not yet been taken into account in our modelling of diffraction peaks, it is obvious from Fig. 4b that it is important. We shall here first rule out the effect of interface dislocations, then consider the effect of dislocations moving within the $\gamma^{\prime}$ rafts on the distribution of elastic strains within the material. (As dislocations are more mobile in the corridors than in the rafts, their densities should be much lower).

The highly distorted areas in the vicinity of the cores of interface dislocations should strongly contribute to the peak tails, at distances d lower than half the distance between these dislocations, i.e. $\mathrm{d}<5.10^{-8} \mathrm{~m}$. The corresponding strain is $b / 2 \pi d=3 \cdot 10^{-2}$, more than five times larger than the distance between the $\gamma$ and $\gamma^{\prime}$ peaks. At scales intermediate between $\mathrm{d}$ and the

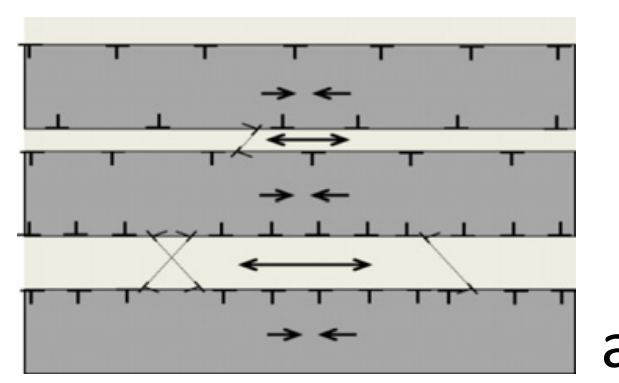

a

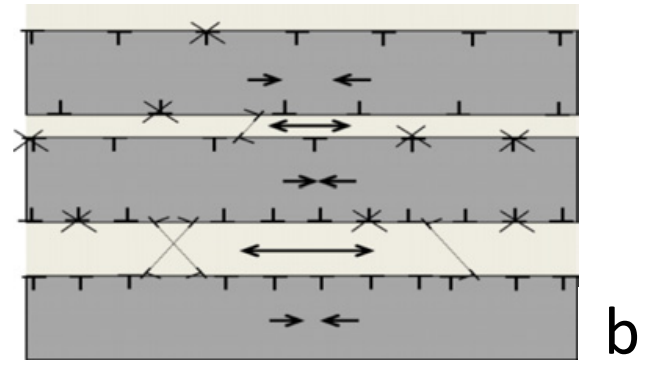

Figure 6. Homogeneity and heterogeneity of the interface dislocation contribution to the $\sigma_{x x}$ component of the stress tensor, before and after the onset of plasticity within the rafts.

corridor thickness, we may expect fluctuations of the elastic strain which decrease as the distance $\mathrm{d}_{i}$ to the interface increases. The decrease is exponential for an infinite periodic dislocation wall [10]. If we consider disordered walls, the variance of the strain fluctuations may decrease as $(\mathrm{d} / \mathrm{di})^{-1}$ or $(\mathrm{d} / \mathrm{di})^{-3}$ depending on the type of disorder $[11,12]$. As the rafts are thicker than the corridors, the contribution of these fluctuations is probably larger in the case of the $\gamma$ peak: the slope of the W' vs. W plot Fig. 3 should be smaller than one. The fluctuations should also be larger when the disorder is high, when new dislocations are added randomly at the interfaces, i.e. when the strain rate of the corridors is high. They might then smooth out when the strain rate decreases again, and interface dislocations may rearrange into a more orderly, low energy way. The evidence for such transient peak width increase is scarce in Fig. 5.

Several arguments support a link between dislocations within the $\gamma^{\prime}$ phase and the reversible widening of the peaks:

- Both peaks thicken when the strain rate of the rafts is high.

- Dislocation densities observed by TEM (Fig. 5c) are near $10^{13} \mathrm{~m}^{-2}$ when the experiment is stopped at high strain rates for large values of $\mathrm{W}$ and $\mathrm{W}$ ', and $10^{12} \mathrm{~m}^{-2}$ or lower when $\mathrm{W}$ and $\mathrm{W}$ ' were allowed to relax under low stress.

- The slope of the W' vs. W plots is higher than one: as the largest fluctuations occur near the dislocation cores, the $\gamma$ corridors are shielded.

An attempt to model the evolution of the distribution of elastic strains with the dislocation density using a $2 \mathrm{D}$ simulation is shown in Fig. 7. A $2 \mathrm{D}$ supercell $20^{2} \mu \mathrm{m}^{2}$ wide containing a stack of $40 \gamma-\gamma^{\prime}$ periods with a lognormal size distribution and a 0.6 fraction of $\gamma^{\prime}$ was built. Different densities $\rho_{d}$ ' of edge dislocations with a 

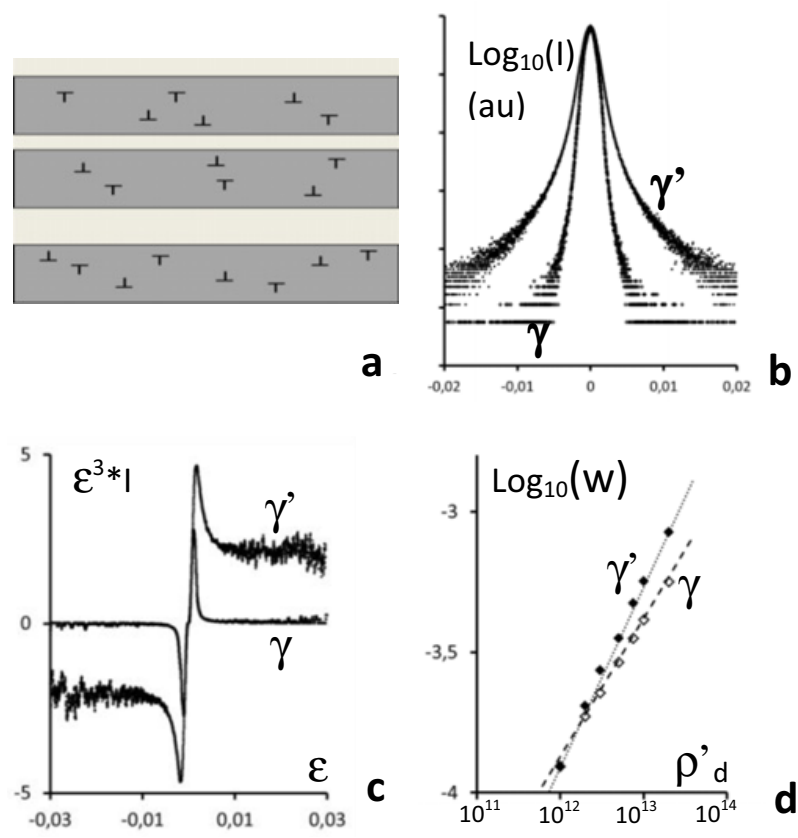

Figure 7. 2D modelling of the distribution of elastic strains due to dislocation within the $\gamma^{\prime}$ rafts. a) Model; b) strain distribution in both phases; c) plot of $\varepsilon^{3} . \mathrm{I}(\varepsilon)$ vs. $\varepsilon$; d) evolution of the W parameters with the dislocation density within the rafts.

[100] Burgers vectors at random positions (but the same number of positive and negative signs in each raft) were added in the $\gamma^{\prime}$ rafts. The distribution of strains was calculated for an isotropic and homogenous material using periodic conditions, and is given for both phases in Fig. 7b for $\rho_{d}{ }^{\prime}=10^{13}$. As expected, the $\gamma^{\prime}$ peak is wider and has a larger tail: the plot of $\varepsilon^{3} . \mathrm{I}(\varepsilon)$ in c) shows that the tails of the distribution indeed varies as $\varepsilon^{-3}[13,14]$ in the $\gamma^{\prime}$ phase for strains larger than 0.01 , and decreases faster in the $\gamma$ phase.

The double logarithmic plot in Fig. 7d shows that $\mathrm{W}$ and 'W' vary as power laws of $\rho_{d}$ ' with exponents $0.49(\mathrm{~W})$ and 0.64 (W'). From statistical arguments (the variance of $\mathrm{W}$ and $\mathrm{W}$ ' should vary as the dislocation density), a 0.5 exponent is expected. Last, the shape of a diffraction peak does not depend only on the distribution of strain, but also on the size of coherent zones, which cannot be modelled in the present approximation.

\section{Conclusion}

From the present examples, it can be seen that high resolution diffraction peaks can give much more data on a superalloy than just peak positions. The peaks are very sensitive to the details of the microstructure (bevelled cubes) and the distribution of elastic strains within the microstructure. Thus, in situ diffraction can become a very powerful tool to test the results of models of the plastic behaviour of superalloys.

However, a full analysis of those peaks will require extensive mechanical and physical modelling of the material, taking many effects into account: elastic anisotropy and inhomogeneity, point defects, and most of all dislocations: further work is needed.

The authors would like to thank the ESRF (ID15A beamline) and DESY (P07 beamline, "CALIPSO: EU Support of Access to Synchrotrons/FELs in Europe") for beamtime and help for the experiments, and Snecma (SAFRAN) for providing the AM1 specimens.

\section{References}

[1] P. Caron and T. Khan, Materials Science and Engineering 61, (1983) 173

[2] T.M. Pollock and A.S. Argon, Acta Metall. Mater 40, (1992) 1

[3] F. Mompiou and D. Caillard, Mater. Sci. Eng. A 483, (2008) 143

[4] K. D. Liss et al., J. Synchrotron Rad. 5, (1998) 82

[5] N. Vaxelaire et al, New Journal of Physics 12, 035018 (2010)

[6] T. Mura, Micromechanics of defects in solids $2^{\text {nd }}$ edition, Martinus Nijhof editions (1987), ISBN 90247-3343-X

[7] H. Moulinec, P. Suquet, Comp. Meth. Appl. Mech. Engng. 157, 69-94 (1998)

[8] H. Mughrabi, H. Biermann and T. Ungar, Journal of Material Engineering and Performance 2(4), 557 (1993)

[9] L. Dirand et al., Phil. Mag. 93 (2013) 1384

[10] J.P. Hirth and J. Lothe, Theory of dislocations, 2nd New edition of Revised edition, Krieger Publishing Company (1991) ISBN-13: 978-0894646171

[11] G. Saada and E. Bouchaud, Acta Metall. 41, (1993) 2173

[12] G. Saada and D. Sornette, Acta Metall. 43, (1995) 313

[13] T. Ungár, and A. Borbély, Applied Physics Letters 69, (21), (1996) 3173

[14] I. Groma, and A. Borbély, A. Diff. Analysis of the Microst. of Materials 68 (2004) 287 